\title{
Study of Membrane Proteins by Single Particles Electron Microscopy Using Detergent, Liposomes and Nanodiscs
}

\author{
Lucien Fabre ${ }^{1}$, Driss Mountassif ${ }^{1}$, Huan Bao $^{2}$, Franck Duong ${ }^{2}$, Isabelle Rouiller ${ }^{1}$ \\ ${ }^{1}$ Department of Anatomy and Cell Biology, McGill University, Montreal, QC, Canada; \\ ${ }^{2}$ Department of Biochemistry \& Molecular Biology, University of British Columbia, 2350 Health \\ Sciences Mall, Vancouver, BC V6T 1Z3, Canada
}

Membrane proteins represent between 20 and 30 percent of encoded protein in most genomes. Their functions are capital for the cell surviving and are the target of more than $50 \%$ of the modern drugs. However, their structural studies remain challenging. Indeed, less than $1 \%$ of the protein structures published in Protein Data Bank are membrane proteins. The main limitation is crystallization difficulty. Meanwhile, single particle Electron Microscopy (SP-EM) is an alternative method especially for large membrane protein complexes. Using SP-EM, membrane complexes can be studied solubilised with detergent but also in membrane mimic systems such as liposomes and nanodiscs.

We have studied two different membrane protein complexes using these different approaches, namely the anthrax toxins and the bacterial maltose transporter MalFGK.

The anthrax toxins (secreted by Bacillus anthracis) are the best characterized prototypes of the family of AB toxins (or binary toxins). They are assembled from three proteins; a single B protein, the protective antigen $(\mathrm{PA} 83 ; 83 \mathrm{kDa})$ that serves as a delivery vehicle for two A proteins, the lethal factor (LF; 90 $\mathrm{kDa})$, and the edema factor $(\mathrm{EF} ; 89 \mathrm{kDa})$. After cleavage by the protease furin of PA to a $63 \mathrm{kDa}$ form (PA63), PA63 assembles into a soluble heptameric prepore conformation (PA63h). After binding to cellular receptors, PA63h is endocytosed. The reduction of $\mathrm{pH}$ in the endosomal compartment is thought to trigger conversion of PA63h from a prepore to a pore conformation somehow allowing LF and EF to reach the cytosol. The conversion from the soluble prepore conformation to the insoluble pore conformation can be reproduces in vitro by lowering the $\mathrm{pH}$. We studied the pore conformation of PA63h in the presence of detergent, liposomes and nanodiscs (figure 1). The results obtained with the different systems will be presented.

The maltose transport (MalFGK) is a prototype of the ATP-binding cassette transporters. MalFGK couples ATP hydrolysis to maltose substrate transport across the cell membrane. It has been shows that the membrane environment is important for proper coupling between conformational changes, ATP hydrolysis and transport. In order to maintain this membrane environment, we studied MalFGK in the nanodiscs system. Nanodiscs consist of a small portion of membrane surrounded by an amphiphatic or scaffold protein. Our preliminary results show that the 3D structure of the MalFGK complex is well preserve in the nanodiscs system even when embedded in negative stain (figure 2).

We will discuss the efficacy of the different systems (detergent, liposomes and nanodiscs) to study membrane protein complexes in single particle electron microscopy. These different methods are part of a powerful toolbox to study membrane proteins, and different biological systems may favor the use of one or several of these techniques. 
a

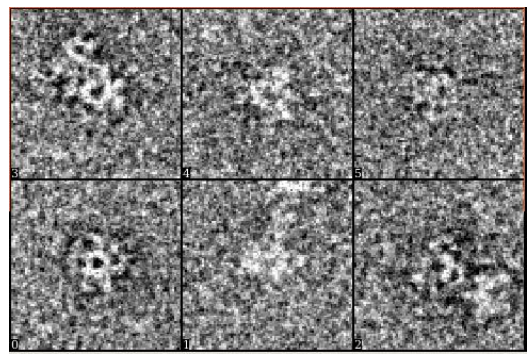

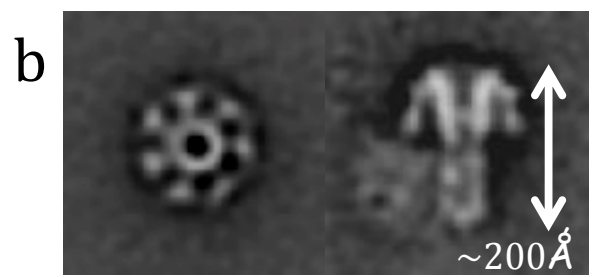

C

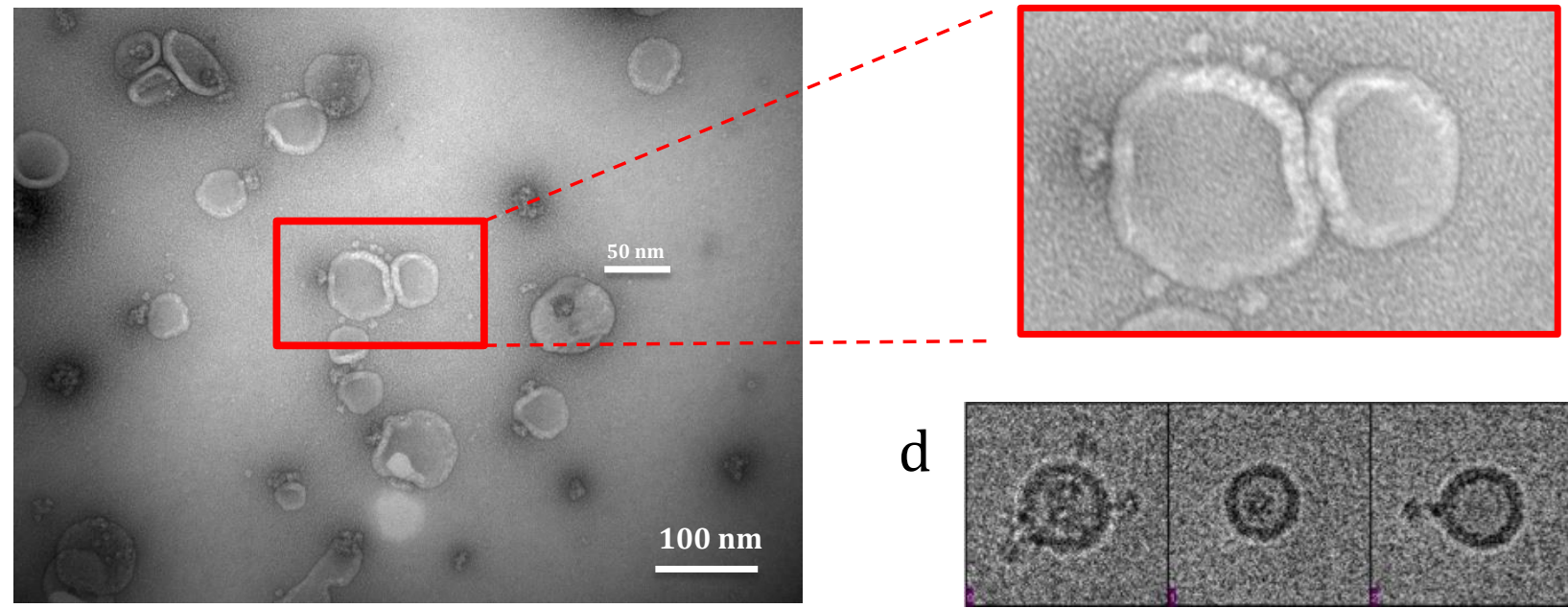

Figure 1: Anthrax Protective Antigen in pore conformation. a) PA-pore single particles stabilized with DDM; b) 2D averages: c) Negative Stain digital micrograph of PA-pore inserted into liposomes; d) Isolated expanded nanodiscs containing PA-pore imaged by cryo-EM; e) Filtered at 20 Á resolution.
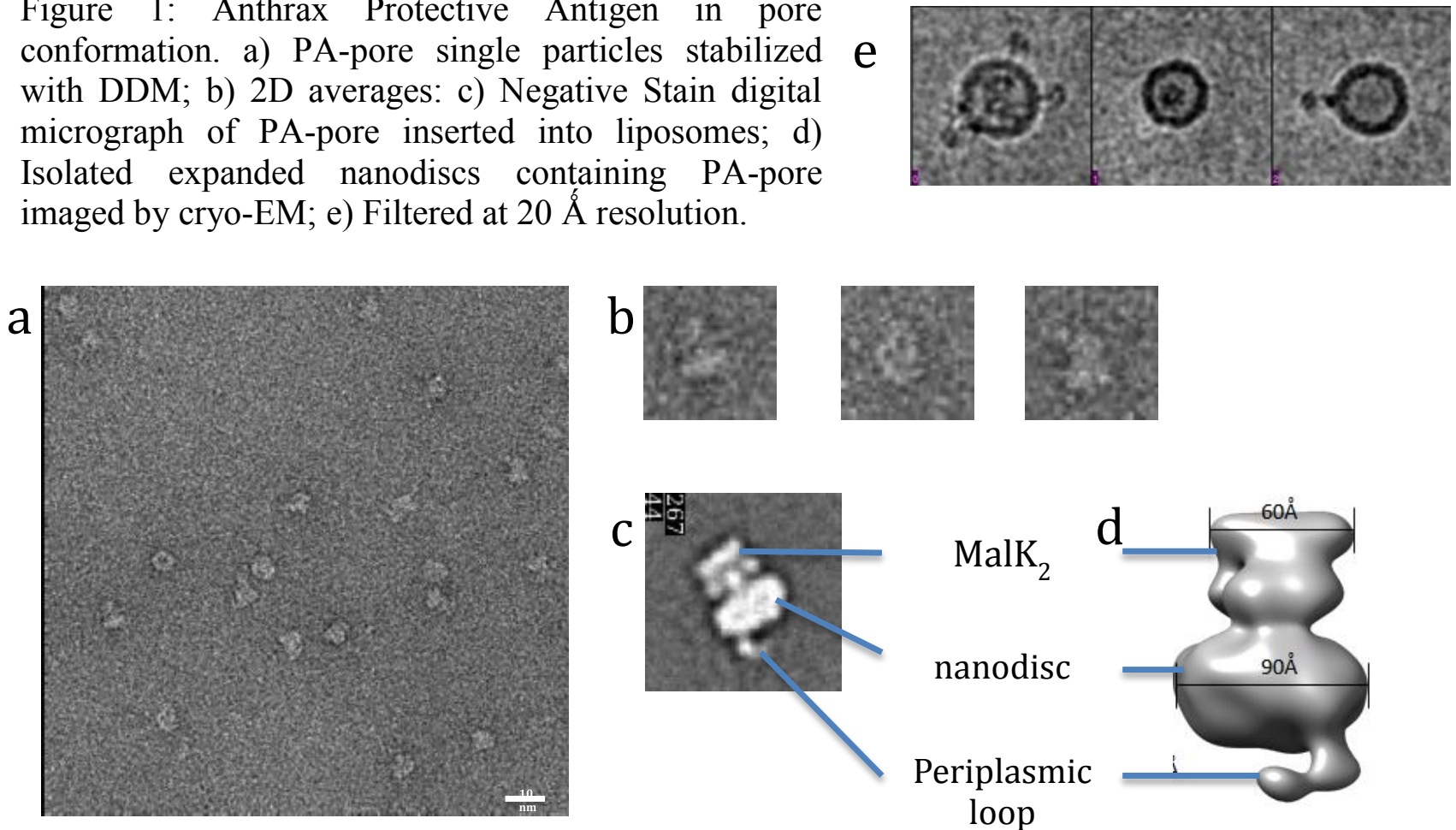

Figure 2: MalFGK transporter complex inserted into nanodiscs. a) Negative Stain EM digital micrograph; b) Isolated particles; c) 2D average of a side view; d) Isosurface of the complex 3D reconstruction. 